\title{
Path integral in area tensor Regge calculus and complex connections
}

\author{
V.M. Khatsymovsky \\ Budker Institute of Nuclear Physics \\ Novosibirsk, 630090, Russia \\ E-mail address: khatsym@inp.nsk.su
}

\begin{abstract}
Euclidean quantum measure in Regge calculus with independent area tensors is considered using example of the Regge manifold of a simple structure. We go over to integrations along certain contours in the hyperplane of complex connection variables. Discrete connection and curvature on classical solutions of the equations of motion are not, strictly speaking, genuine connection and curvature, but more general quantities and, therefore, these do not appear as arguments of a function to be averaged, but are the integration (dummy) variables. We argue that upon integrating out the latter the resulting measure can be well-defined on physical hypersurface (for the area tensors corresponding to certain edge vectors, i.e. to certain metric) as positive and having exponential cutoff at large areas on condition that we confine ourselves to configurations which do not pass through degenerate metrics.
\end{abstract}


The functional integral approach remains the most efficient method of quantization of such the discrete version of general relativity as Regge calculus [1] (RC). General conception of this approach, definitions and theorems were discussed in [2]. Functional integral is a basic tool in investigation of the properties of quantum $\mathrm{RC}[3,4]$ (one of the consequences of this study being occurrence of the phase transitions in simplicial quantum gravity). For a review of $\mathrm{RC}$ and alternative discrete gravity approaches see, e. g., [5].

Functional integral in the continuum theory is usually defined as Feynman path integral based on the canonical (Dirac) quantization prescription. Since continuous time coordinate is absent in RC, now we cannot develop Hamiltonian formalism and canonical quantization. So we are free to choose another physical requirements to hopefully fix completely discrete functional integral measure, and different sets of these requirements do not necessarily result in the same measure. Standard choice of the measure was considered in [4]. The choice of interest for us (a posteriori motivated by the hopes to get absolutely convergent functional integral) is to require that some limiting continuous time form of the functional integral of interest be exactly Feynman path integral in the limiting continuous time (so-called $(3+1)$ ) RC.

The present paper continues our work on studying the functional integral defined in such the way. Let us briefly summarize main points in the solution to this problem. A coordinate is made continuous by shrinking sizes of all the simplices along this coordinate to those infinitely close to zero. Then one regards this coordinate as a formal time and tries to develop Hamiltonian formalism and canonical quantization and represent the result in the form of the Feynman path integral measure.

Next it is natural to ask whether a measure in the original completely discrete RC exists such that one could define the formal limit of this measure when one of the coordinates is made continuous and the limiting measure would coincide with the above Feynman path (canonical quantization) measure with this continuous coordinate playing the role of time. Equivalence of the different coordinates means that this situation should take place irrespectively of what coordinate is made continuous.

A difficulty with $\mathrm{RC}$ in the continuous time limit is that the description of the infinitely flattened in some direction simplex purely in terms of the lengths is singular. The idea is to use description in terms of the variables of the types of both lengths and angles. This might be achieved in the Regge analog of the Hilbert-Palatini form of the Einstein action. The discrete analogs of the tetrad and connection, edge vectors and finite rotation matrices, were first considered in [2]. Approximate representation (for small deficit angles) of the Regge action in terms of edge vectors and independent finite rotation matrices has been proposed in the paper [6]. Simultaneously, we have proposed in our paper [7] representations (ordinary and selfdual ones) of the Regge action for arbitrary deficit angles. The representation of this type results in the exact Regge action if rotation matrices are excluded via equations of motion. Rotation matrices are just the desired angle type variables which allow us to formulate continuous time $(3+1) \mathrm{RC}$ in a nonsingular way. After that the above strategy can be implemented. In [8] we write out canonical form of $\mathrm{RC}$ in the considered variables.

Next we can try to solve the problem of finding measure in the full discrete $\mathrm{RC}$ 
which has the desired continuous time limit corresponding to the canonical quantization irrespectively of what coordinate is taken as a time and made continuous. Although this last condition is rather restrictive, the problem has solution in 3 dimensions [9]. In 4 dimensions solution can be found for a certain version of the so-called "area RC" where areas are treated as independent variables [10,5]. Since the number of areas is larger than the number of lengths, this means that the lengths of the same edge defined in the different simplices are in general different, i.e. ambiguous. The configuration superspace of the area RC contains the hypersurface corresponding to the ordinary RC; at the same time it is exactly soluble just as 3D model. Appropriate version in our case is "area tensor RC" with independent area tensors (i.e., in particular, in general there are no edge vectors corresponding to them). Just the corresponding superspace (extended as compared to that of ordinary RC) is that space on which the measure under above restrictive conditions can be found [11].

Remarkable feature simplifying solution of the above problem of finding full discrete measure for the 3D RC and for the $4 \mathrm{D}$ area tensor $\mathrm{RC}$ in the tetrad-connection variables is commutativity of the constraints (arising in the Hamiltonian formalism, i.e. in the continuous time limit). These constraints as well as canonical quantization itself are analogous to their completely continuum counterparts. The commuting constraints for the 3D discrete gravity were found in [12] for general system (not a'priori restricted to be $\mathrm{RC}$ ). Analogous first class system of constraints arises in the area tensor RC in the above mentioned derivation of the measure [11].

Finally, we need to reduce the quantum measure in the extended superspace to the RC hypersurface. The idea is to consider area tensor RC system and ordinary RC system as particular case of the simplicial complex with discontinuous metrics. The point is that the piecewise flat manifold possesses metric whose normal component undergoes discontinuity when passing across any $3 \mathrm{D}$ face, but the tangential components remain unchanged. Now we go further and consider system where tangential components of metric are also discontinuous. It is the system with independent simplices which do not necessarily fit each other on their common faces. In the superspace of all the simplicial discontinuous metrics RC corresponds to the hypersurface singled out by conditions of the tangential metric continuity on the faces. The problem is to reduce the above constructed quantum measure in "area tensor RC" to this hypersurface. For that some $\delta$-functionlike factor is introduced in the measure which fixes equality of tangential metric across any face. This factor is found in our paper [13] by using the principle of "minimum of the lattice artefacts". Namely, we require that the factor should not depend on the form and size of any face across which metrics are compared, only on the hyperplane in which the given face is placed. We show that such the factor preserving equivalence of the different simplices exists and is unique. Consequences of the viewpoint on area RC (now not tensor one) as a system with discontinuous metrics were also discussed in [14].

The result of [11] for the Euclidean measure being applied to arbitrary function on the set of area tensors $\pi_{\sigma^{2}}^{a b}$ and connection matrices $\Omega_{\sigma^{3}}^{a b}$ reads

$$
<\Psi(\{\pi\},\{\Omega\})>=\int \Psi(-i\{\pi\},\{\Omega\}) \exp \left(-\sum_{t-\text { like } \sigma^{2}} \tau_{\sigma^{2}} \circ R_{\sigma^{2}}(\Omega)\right)
$$




$$
\begin{gathered}
\times \exp \left(i \sum_{\text {not } t-\text { like } \sigma^{2}} \pi_{\sigma^{2}} \circ R_{\sigma^{2}}(\Omega)\right) \prod_{\substack{\text { not } \\
t-\text { like } \\
\sigma^{2}}} \mathrm{~d}^{6} \pi_{\sigma^{2}} \prod_{\sigma^{3}} \mathcal{D} \Omega_{\sigma^{3}} \\
\equiv \int \Psi(-i\{\pi\},\{\Omega\}) \mathrm{d} \mu_{\text {area }}(-i\{\pi\},\{\Omega\}) .
\end{gathered}
$$

Here $A \circ B \stackrel{\text { def }}{=} \frac{1}{2} A^{a b} B_{a b}, \mathcal{D} \Omega_{\sigma^{3}}$ is the Haar measure on the group $\mathrm{SO}(4)$ of connection matrices $\Omega_{\sigma^{3}}$, the curvature $R_{\sigma^{2}}(\Omega)$ is path-ordered product of connections $\Omega_{\sigma^{3}}$ along the path enclosing the triangle $\Omega_{\sigma^{2}}$. Here rotation of the integration contours used to define integral is performed via substitution of the integration variables $\pi_{\sigma^{2}} \rightarrow-i \pi_{\sigma^{2}}$. The $\pi_{\sigma^{2}}$ and $\tau_{\sigma^{2}}$ are the area tensors for the "not t-like" and "t-like" triangles, respectively, while arbitrary tensor $\left(\pi_{\sigma^{2}}\right.$ or $\left.\tau_{\sigma^{2}}\right)$ will be denoted $v_{\sigma^{2}}$. The definitions of these triangle are intuitively understandable, the notion "t-like" should refer to a triangle (or, more generally, to a simplex of arbitrary dimensionality) with one of the edges connecting the pair of corresponding points in the two analogous in structure neighbouring leaves of the foliation (3D Regge structures themselves) along one of the coordinates named time t. The usual definition "timelike" is reserved to be attribute of the local frame indices $a$, $b$ in $v_{\sigma^{2}}^{a b}$ while the above "t-like" refers to $\sigma^{2}$, the Regge analog of the pair of the world indices.

The formula (1) looks as the usual field-theoretical path integral expression with exception of the following three points. First, occurrence of the $\mathrm{SO}(4)$ Haar measure $\mathcal{D} \Omega_{\sigma^{3}}$ instead of the Lebesgue measure on the infinitesimal connections in the continuum case. This is connected with the specific form of the kinetic term $\pi_{\sigma^{2}} \circ \Omega_{\sigma^{2}}^{\dagger} \dot{\Omega}_{\sigma^{2}}$ obtained when we are going over to the continuous time limit (here $\Omega_{\sigma^{2}}$ serves to parameterize limiting form of $\Omega_{\sigma^{3}}$ with $\sigma^{3}$ filling up the infinitesimal t-like 3-prism with the base $\sigma^{2}$ ). Canonical quantization of the theory with such the kinetic term just gives $\mathcal{D} \Omega_{\sigma^{2}}$. Tracing back to the original full discrete theory, this means occurrence of $\mathcal{D} \Omega_{\sigma^{3}}$ there too.

Second, there are the terms $\pi \circ R$ in the exponential instead of the Regge action which in the exact connection representation would be the sum of the terms with 'arcsin' of the type $|\pi| \arcsin (\pi \circ R /|\pi|)[7]$. The reason is that the exponential should be the sum of the constraints times Lagrange multipliers in order to fit the canonical quantisation prescription in the continuous time limit whatever coordinate is taken as a time. These constraints just do not contain 'arcsin' (in empty spacetime; situation is more complicated in the presence of matter fields!).

Third, absence of integrations over some set of area tensors $\tau_{\sigma^{2}}$. Formally, this looks like the gauge fixing of some tetrad/area components in the continuous time $(3+1) \mathrm{RC}$ or in the continuum general relativity (and indeed reduces to that gauge fixing in the continuous time limit $[9,11])$. In the fully discrete theory the reason for fixing $\tau_{\sigma^{2}}$ is dependence of the curvature matrices on the t-like triangles on all the rest curvatures via Bianchi identities. It is the set of those triangles the curvature matrices on which are functions, via Bianchi identities, of all the rest curvatures. Integrations over $\tau_{\sigma^{2}}$ might result in the singularities of the type of $[\delta(R-\bar{R})]^{2}$. In the situation when one defines direction of the coordinate called "time", the $t$-like triangles turn out to be possible choice of the above set. One could keep in mind a regular way of constructing 4D Regge geometry of the 3D leaves, arbitrary 3D Regge geometries themselves. For general 4D 
Regge geometry we adopt the general definition of the set of "t-like triangles" as the triangles carrying dependent (via Bianchi identities) curvature matrices. In the present paper we study Regge geometry where"t-like triangles" will be defined just in this way.

Brief discussion of the above points is given in our work [15]. Expressions like (1) present possible exact definition of the formal path integral symbol in general relativity.

Consider Regge lattice composed of the two identical, up to reflection, 4-simplices with the vertices $0,1,2,3,4$. We can imagine this complex if consider the two 4-simplices (01234) and (0'1234) with common 3-face (1234) and identify the vertices 0 and $0^{\prime}$. Let all the connections $\Omega$ on the 3 -faces act from (01234) to $\left(0^{\prime} 1234\right)$, that is, if a 2 -face tensor $\pi$ is defined in (01234), then $\Omega \pi \bar{\Omega}$ is defined in $\left(0^{\prime} 1234\right)$. Denote a 3 -face in the same way as the opposite vertex, $\Omega_{i} \equiv \Omega_{\sigma^{3}}$ where $\sigma^{3}=(\{01234\} \backslash\{i\})$. Here $\{\ldots\}$ denote (sub)set, here of the vertices $0,1,2,3,4$. Denote a 2 -face in the same way as the opposite edge, $v_{(i k)} \equiv v_{\sigma^{2}}, R_{(i k)} \equiv R_{\sigma^{2}}$ where $\sigma^{2}=(\{01234\} \backslash\{i k\})$. It is convenient to define the variables $v, R$ on the ordered pairs of vertices $i k, v_{i k}=-v_{k i}, R_{i k}=\bar{R}_{k i}$. (Then $v_{(i k)}$ is a one of the two values, $v_{i k}$ or $v_{k i}, R_{(i k)}$ is $R_{i k}$ or $R_{k i}$ ). The action takes the form

$$
S(v, \Omega)=\sum_{i<k}^{4}\left|v_{i k}\right| \arcsin \frac{v_{i k} \circ R_{i k}}{\left|v_{i k}\right|}, \quad R_{i k}=\bar{\Omega}_{i} \Omega_{k} .
$$

The curvature matrices are related by the Bianchi identities $R_{i k} R_{k l}=R_{i l}$ (on the triangles with common edge $(\{01234\} \backslash\{i k l\}))$. As independent curvature matrices we can choose $R_{\alpha} \equiv R_{0 \alpha}(\alpha, \beta, \gamma, \ldots=1,2,3,4)$, that is, the curvature on the 2 -faces of the tetrahedron (1234). Thereby, in accordance with the above definition, the tetrahedron (1234) can be attributed to the leaf of the foliation (is not t-like) while the other triangles $(0 \alpha \beta)$ can be treated as $t$-like ones. This corresponds to the naive viewpoint on this Regge lattice as that one corresponding to the evolution in time of the closed manifold consisting of the two copies of the tetrahedron (1234) to the point 0. Upon dividing the triangles into the $t$-like ones with the tensors $\tau_{\alpha \beta} \equiv v_{\alpha \beta}$ entering as parameters and the leaf ones with the tensors $\pi_{\alpha} \equiv v_{0 \alpha}$ the action reads

$$
S(v, \Omega)=\sum_{\alpha=1}^{4}\left|\pi_{\alpha}\right| \arcsin \frac{\pi_{\alpha} \circ R_{\alpha}}{\left|\pi_{\alpha}\right|}+\sum_{\alpha<\beta}^{4}\left|\tau_{\alpha \beta}\right| \arcsin \frac{\tau_{\alpha \beta} \circ\left(\bar{R}_{\alpha} R_{\beta}\right)}{\left|\tau_{\alpha \beta}\right|} .
$$

Due to invariance property of the Haar measure the product of $\mathcal{D} \Omega_{\sigma^{3}}$ over all $\sigma^{3}$ can be expressed as the product of $\mathcal{D} R_{\sigma^{2}}$ over all not t-like $\sigma^{2}$ (i.e. $R_{\sigma^{2}}$ on these $\sigma^{2}$ are independent) and $\mathcal{D} \Omega_{\sigma^{3}}$ over some set of $\sigma^{3}$. In the considered case we can choose

$$
\prod_{i=0}^{4} \mathcal{D} \Omega_{i}=\mathcal{D} \Omega_{0} \prod_{\alpha=1}^{4} \mathcal{D} R_{\alpha}
$$

Integration over $\mathcal{D} \Omega_{0}$ is trivial, and our expression for the vacuum expectation value takes the form

$$
\begin{gathered}
<\Psi(\{\pi\},\{R\})>=\int \Psi(-i\{\pi\},\{R\}) \exp \left[-\sum_{\alpha<\beta}^{4} \tau_{\alpha \beta} \circ\left(\bar{R}_{\alpha} R_{\beta}\right)\right] \\
\times \exp \left(i \sum_{\alpha=1}^{4} \pi_{\alpha} \circ R_{\alpha}\right) \prod_{\alpha=1}^{4} \mathrm{~d}^{6} \pi_{\alpha} \prod_{\alpha=1}^{4} \mathcal{D} R_{\alpha} .
\end{gathered}
$$


Since curvature matrices on classical solutions are not quite physical ones (are more general than rotations by defect angle around triangles $\sigma^{2}$ ), it is natural to limit ourselves by considering functions to be averaged of purely tensors $\pi_{\sigma^{2}}$. More accurately, matrices $R$ whenever appearing among arguments of a function to be averaged should be substituted by their exact expressions in terms of $\pi_{\sigma^{2}}$. Let us perform integration of the measure over curvature matrices. To this end, we decompose antisymmetric tensor variables into self- and antiselfdual parts as

$$
v_{a b} \equiv \frac{1}{2}{ }^{+} v_{k}{ }^{+} \Sigma_{a b}^{k}+\frac{1}{2}{ }^{-} v_{k}-\Sigma_{a b}^{k}
$$

where ${ }^{ \pm} \sum_{a b}^{k}$ is basis of (anti)selfdual matrices such that $i^{ \pm} \Sigma_{a b}^{k}$ obey Pauli matrix algebra. The curvature splits into factors

$$
{ }^{ \pm} R=\exp \left({ }^{ \pm} \boldsymbol{\phi}^{ \pm} \boldsymbol{\Sigma}\right)=\cos { }^{ \pm} \phi+{ }^{ \pm} \boldsymbol{\Sigma}^{ \pm} \boldsymbol{n} \sin { }^{ \pm} \phi,
$$

$\mathrm{SU}(2)$-rotations by the angles ${ }^{ \pm} \phi$ around unit vectors ${ }^{ \pm} \boldsymbol{n}={ }^{ \pm} \phi /{ }^{ \pm} \phi$ in the corresponding 3D spaces. In the particular physical case when $R_{\sigma^{2}}$ rotates around $\sigma^{2}$ by the defect angle on $\sigma^{2}$, both ${ }^{ \pm} \phi_{\sigma^{2}}$ are equal to the half of that defect angle.

The measure splits multiplicatively into the factors in the configuration spaces of selfand antiselfdual tensor variables,

$$
\begin{aligned}
\mathrm{d} \mu_{\text {area }} & =\mathrm{d}^{+} \mu_{\text {area }} \mathrm{d}^{-} \mu_{\text {area }}, \quad \mathrm{d}^{ \pm} \mu_{\text {area }} \equiv \mathrm{d}^{ \pm} \mathcal{N} \prod_{\alpha=1}^{4} \mathrm{~d}^{3}{ }^{ \pm} \boldsymbol{\pi}_{\alpha}, \\
\mathrm{d}^{ \pm} \mathcal{N} & =\exp \left[-\sum_{\alpha<\beta}{ }^{ \pm} \tau_{\alpha \beta} \circ\left({ }^{ \pm} R_{\alpha}{ }^{ \pm} R_{\beta}\right)-\sum_{\alpha=1}^{4}{ }^{ \pm} \pi_{\alpha} \circ{ }^{ \pm} R_{\alpha}\right] \prod_{\alpha=1}^{4} \mathcal{D}^{ \pm} R_{\alpha} .
\end{aligned}
$$

In what follows, indices \pm on the variables will be suppressed where it is possible without confusion, and we shall have in view any one of sectors, self- or antiselfdual one. In the formal limit $\tau_{\alpha \beta}=0$ this measure (as well as the general one (1)) can be factorized into the measures on separate pairs ${ }^{ \pm} \pi_{\alpha},{ }^{ \pm} R_{\alpha}$. We can apply any such factor to averaging monomials of the variables and then extend the result to arbitrary functions like $f\left(\pi_{\alpha}^{2}\right)$,

$$
\begin{aligned}
& \int \exp \left(i \boldsymbol{\pi}_{\alpha} \boldsymbol{n}_{\alpha} \sin \phi_{\alpha}\right) \frac{\sin ^{2} \phi_{\alpha}}{\phi_{\alpha}^{2}} \mathrm{~d}^{3} \boldsymbol{\phi}_{\alpha} \mathrm{d}^{3} \boldsymbol{\pi}_{\alpha} f\left(-\pi_{\alpha}^{2}\right)= \\
& \quad \int_{-\infty}^{+\infty} \cosh ^{2} \eta_{\alpha} \mathrm{d} \eta_{\alpha} \int_{0}^{\infty} 2 \pi \sinh \zeta_{\alpha} \mathrm{d} \zeta_{\alpha} \int \mathrm{d}^{3} \boldsymbol{\pi}_{\alpha} \exp \left(-\pi_{\alpha} \cosh \eta_{\alpha} \cosh \zeta_{\alpha}\right) f\left(\pi_{\alpha}^{2}\right) .
\end{aligned}
$$

The LHS is the above " $\pi_{\sigma^{2}} \rightarrow-i \pi_{\sigma^{2}}$ "-definition of the Euclidean integral

$$
\int \exp \left(-\boldsymbol{\pi}_{\alpha} \boldsymbol{n}_{\alpha} \sin \phi_{\alpha}\right) \frac{\sin ^{2} \phi_{\alpha}}{\phi_{\alpha}^{2}} \mathrm{~d}^{3} \boldsymbol{\phi}_{\alpha} \mathrm{d}^{3} \boldsymbol{\pi}_{\alpha} f\left(\pi_{\alpha}^{2}\right),
$$

while RHS looks as a result of the formal substitution

$$
\phi_{\alpha}=\pi / 2+i \eta_{\alpha}, \quad \theta_{\alpha}=i \zeta_{\alpha}
$$


in (10), $\theta_{\alpha}$ is the angle between $\phi_{\alpha}$ and $\boldsymbol{\pi}_{\alpha} ; \eta_{\alpha}, \zeta_{\alpha}$ are real. Let us integrate over contours (11) in the case $\tau_{\alpha \beta} \neq 0$ of interest. The formula (8) gives

$$
\begin{array}{r}
{ }^{ \pm} \mathcal{N} \equiv \int \mathrm{d}^{ \pm} \mathcal{N}=\int \exp \left[-\sum_{\alpha=1}^{4} \pi_{\alpha} z_{\alpha} \cosh \eta_{\alpha}+\sum_{\alpha<\beta}^{4} i \boldsymbol{\tau}_{\alpha \beta} \cdot\left(\boldsymbol{n}_{\alpha} \cosh \eta_{\alpha} \sinh \eta_{\beta}-\right.\right. \\
\left.\left.-\boldsymbol{n}_{\beta} \cosh \eta_{\beta} \sinh \eta_{\alpha}\right)+\sum_{\alpha<\beta}^{4} \boldsymbol{\tau}_{\alpha \beta} \cdot\left(\boldsymbol{n}_{\alpha} \times \boldsymbol{n}_{\beta}\right) \cosh \eta_{\alpha} \cosh \eta_{\beta}\right] \prod_{\alpha=1}^{4} \cosh ^{2} \eta_{\alpha} \mathrm{d} \eta_{\alpha} \mathrm{d} z_{\alpha} \mathrm{d} \chi_{\alpha}
\end{array}
$$

where $z_{\alpha}=\cosh \zeta_{\alpha}, \chi_{\alpha}$ is polar angle of $\phi_{\alpha}$ w. r. t. $\pi_{\alpha}$. Let us write the exponent in (12) in the form $\exp \left(-\sum_{\alpha=1}^{4} \pi_{\alpha} z_{\alpha} \cosh \chi_{\alpha}+f\right)$ and integrate over $\mathrm{d} z_{\alpha}$ by parts, first integrating $\exp \left(-\sum_{\alpha=1}^{4} \pi_{\alpha} z_{\alpha} \cosh \chi_{\alpha}\right)$ and differentiating $\exp f$. We arrive at

$$
\begin{aligned}
& \int_{1}^{\infty} \exp \left(-\sum_{\alpha=1}^{4} \pi_{\alpha} z_{\alpha} \cosh \eta_{\alpha}+f\right) \mathrm{d} z_{\alpha}= \\
&\left.\frac{1}{\pi_{\alpha} \cosh \eta_{\alpha}} \exp \left(-\sum_{\alpha=1}^{4} \pi_{\alpha} z_{\alpha} \cosh \eta_{\alpha}+f\right)\right|_{z_{\alpha}=\infty} ^{z_{\alpha}=1}+O(\tau)
\end{aligned}
$$

The function $f=O(\tau)$. At $\tau_{\alpha \beta} \rightarrow 0$ the value in the RHS of (13) taken at $z_{\alpha}=\infty$ is well-defined and is zero in the range of changing the arguments $\pi_{\alpha}$ tending to the whole range of $\pi_{\alpha}$. The function $f$ is up to $O\left(z_{\alpha}^{-1}\right)$ linear in $z_{\alpha}$, and situation looks like calculation of the integral

$$
\int_{1}^{\infty} \exp (-\lambda z) \mathrm{d} z=\frac{\exp (-\lambda)}{\lambda}
$$

at $\lambda<0$ it is considered as analytical continuation from the region $\lambda>0$. In the case of the formula (13) this means that the value in the RHS taken at $z_{\alpha}=\infty$ should be treated as zero.

Thus, the measure splits into the terms vanishing in the formal limit $\boldsymbol{\tau}_{\alpha \beta}=0$ and an expression taken at $z_{\alpha}=1$. The values $z_{\alpha}=1$ mean that $R_{\alpha}$ rotate around corresponding triangles, i.e. have exact physical sense of curvature matrices. Some disadvantage of our highly nonperturbative model having only one 3D leaf (another one is contracted to the point 0) is that some components $\left|\boldsymbol{\tau}_{\alpha \beta}\right|$ cannot be small on physical hypersurface (when tetrahedrons are closed) if $\pi_{\alpha}$ grow. However, we concentrate on studying just the $z_{\alpha}=1$ term in the RHS of (13) having in view its relevance to the more usual Regge structures where the condition $\left|\boldsymbol{\tau}_{\alpha \beta}\right| \ll 1$ is quite accessible by using closely located and similar in their geometry 3D leaves.

At $z_{\alpha}=1$ integrations over polar angles $\chi_{\alpha}$ are trivial, and we find (up to normalization, as usual)

$$
\begin{gathered}
{ }^{ \pm} \mathcal{N}=\int \exp \left[-\sum_{\alpha=1}^{4} \pi_{\alpha} \cosh \eta_{\alpha}+\sum_{\alpha<\beta}^{4} \boldsymbol{\tau}_{\alpha \beta} \cdot\left(\boldsymbol{n}_{\alpha} \times \boldsymbol{n}_{\beta}\right) \cosh \eta_{\alpha} \cosh \eta_{\beta}\right] \\
\times \cos \left[\sum_{\alpha<\beta}^{4} \boldsymbol{\tau}_{\alpha \beta} \cdot\left(\boldsymbol{n}_{\alpha} \cosh \eta_{\alpha} \sinh \eta_{\beta}-\boldsymbol{n}_{\beta} \cosh \eta_{\beta} \sinh \eta_{\alpha}\right)\right] \prod_{\alpha=1}^{4} \frac{1}{\pi_{\alpha}} \cosh \eta_{\alpha} \mathrm{d} \eta_{\alpha}
\end{gathered}
$$


where from now on $\boldsymbol{n}_{\alpha}=\boldsymbol{\pi}_{\alpha} / \pi_{\alpha}$. Dangerous for convergence is the bilinear in cosh $\eta_{\alpha}$ term in the exponential. What is its sign? It is arbitrary until $\boldsymbol{\tau}_{\alpha \beta}$ are specified, namely, until area tensors are chosen to correspond to certain edge vectors. In this case $\tau_{\alpha \beta}$ are parameterized as follows,

$$
\tau_{\alpha \beta}=u_{\beta} \pi_{\alpha}-u_{\alpha} \pi_{\beta}+\lambda\left[\pi_{\beta}, \pi_{\alpha}\right]
$$

where

$$
\sum_{\alpha=1}^{4} u_{\alpha}=1, \quad \sum_{\alpha=1}^{4} \pi_{\alpha}=0
$$

This anzats solves the conditions on area tensors of the type

$$
v_{\sigma_{1}^{2}} * v_{\sigma_{2}^{2}} \equiv \frac{1}{4} \epsilon_{a b c d} v_{\sigma_{1}^{2}}^{a b} v_{\sigma_{2}^{2}}^{c d}=0
$$

for any two 2-simplices $\sigma_{1}^{2}, \sigma_{2}^{2}$ sharing a common edge, and

$$
v_{\sigma_{1}^{2}} * v_{\sigma_{2}^{2}}= \pm v_{\sigma_{3}^{2}} * v_{\sigma_{4}^{2}}
$$

for any two pairs of 2-simplices of the same 4-simplex such that intersections in these pairs, $\sigma_{1}^{2} \cap \sigma_{2}^{2}$ and $\sigma_{3}^{2} \cap \sigma_{4}^{2}$, are 0 -simplices (vertices). In the present model notations

$$
\pi_{\alpha} * \pi_{\beta}=0, \quad \tau_{\alpha \beta} * \pi_{\alpha}=0, \quad \tau_{12} * \pi_{3}=\tau_{23} * \pi_{1}=\tau_{31} * \pi_{2} .
$$

Irreducible set of 20 constraints follows if $\alpha, \beta$ run over $1,2,3$ here. Self-antiselfdual splitting recasts (16) to vector form,

$$
\boldsymbol{\tau}_{\alpha \beta}=u_{\beta} \boldsymbol{\pi}_{\alpha}-u_{\alpha} \boldsymbol{\pi}_{\beta}+\lambda \boldsymbol{\pi}_{\beta} \times \boldsymbol{\pi}_{\alpha}
$$

so that the sign of the last term in the exponential

$$
\exp \left[-\sum_{\alpha=1}^{4} \pi_{\alpha} \cosh \eta_{\alpha}-\lambda \sum_{\alpha<\beta}^{4}\left(\boldsymbol{n}_{\alpha} \times \boldsymbol{n}_{\beta}\right)^{2} \pi_{\alpha} \pi_{\beta} \cosh \eta_{\alpha} \cosh \eta_{\beta}\right]
$$

is defined by the sign of $\lambda$. Integral over $\mathrm{d} \eta_{\alpha}$ is absolutely convergent at $\lambda \geq 0$ and reminds in this respect

$$
\int_{1}^{\infty} \exp \left(-x-\lambda x^{2}\right) \mathrm{d} x
$$

nonanalytical at $\lambda=0$ and complex if continued to $\lambda<0$. In our case $\lambda=0$ means zero volume of the 4 -simplex, i.e. degenerate metric.

Thus, the measure (15) being reduced to the physical hypersurface (ordinary RC) and integrated over connections can be well-defined on condition that we treat 4-simplices as nondegenerate and preserving orientation. The resulting measure possesses exponential (w. r. t. areas) cutoff. Moreover, on physical hypersurface the resulting full measure proportional to ${ }^{+} \mathcal{N}-\mathcal{N}$ is positive. This is simply because the factors ${ }^{+} \mathcal{N}$ and $-\mathcal{N}$ are equal to the same function of the arguments ${ }^{+} \boldsymbol{\pi}_{\alpha} \cdot{ }^{+} \boldsymbol{\pi}_{\beta},{ }^{+} \boldsymbol{\tau}_{\alpha \beta} \cdot{ }^{+} \boldsymbol{\pi}_{\alpha}$ and ${ }^{-} \boldsymbol{\pi}_{\alpha} \cdot{ }^{-} \boldsymbol{\pi}_{\beta}$, 
${ }^{-} \boldsymbol{\tau}_{\alpha \beta} \cdot{ }^{-} \boldsymbol{\pi}_{\alpha}$, respectively. On physical hypersurface the "+" and "-" counterparts from this set are equal to the same value due to (20) since

$$
v_{1} * v_{2}=\frac{1}{2}{ }^{+} \boldsymbol{v}_{1} \cdot{ }^{+} \boldsymbol{v}_{2}-\frac{1}{2}-\boldsymbol{v}_{1} \cdot{ }^{-} \boldsymbol{v}_{2} .
$$

Therefore $\mathrm{d} \mu_{\text {area }}$ turns out to be proportional to the square of a real value.

We have illustrated by the $\mathrm{RC}$ model of a simple structure that upon going to the complex integration contours in the space of matrices $\Omega$ the quantum measure could be well-defined. Matrices $\Omega_{\sigma^{3}}$ in our formalism are more general values than physical connections since the curvature matrices $R_{\sigma^{2}}$ composed of $\Omega_{\sigma^{3}}$ being classical solution of the equations of motion do not necessarily rotate around the triangle $\sigma^{2}$ (analog of the continuum curvature with torsion). Therefore the curvature matrices entering any physical quantity to be averaged should be considered not as independent ones but as already expressed in terms of area tensors. Then really independent matrices $\Omega_{\sigma^{3}}$ could be integrated out from the very beginning. The resulting measure on physical hypersurface is positive and possesses exponential cutoff at large areas.

The integration contours in the complex connection space pass through independent matrices $R_{\sigma^{2}}$ corresponding to rotations by the angles with real part $\pi / 2$, while the axes of these rotations form the purely imaginary angles with $\sigma^{2}$ area vectors. These contours are strictly separated from trivial curvature matrices and in this sense the analytical continuation could be called nonperturbative one. We have found that up to $O(\tau)$ the integrated over connections quantum measure is defined by its value at the points in the superspace of connections corresponding to independent matrices $R_{\sigma^{2}}$ rotating around triangles $\sigma^{2}$, i.e. around area $\sigma^{2}$ vectors in the self-antiselfdual representation.

The main problem is whether the matrices $R_{\sigma^{2}}$ on the t-like triangles expressed by Bianchi identities in terms of the above complex nonperturbative rotations could provide the contributions $\tau_{\sigma^{2}} \circ R_{\sigma^{2}}$ in the exponential ensuring convergence of the integral over connections. Here we have given affirmative answer for the simplest Regge structure and the more general structures remain to be studied.

The present work was supported in part by the Russian Foundation for Basic Research through Grant No. 05-02-16627-a.

\section{References}

[1] T. Regge, Nuovo Cimento, 19, 558 (1961).

[2] J. Fröhlich, Regge Calculus and Discretized Gravitational Functional Integrals, I. H. E. S. preprint 1981 (unpublished); Non-Perturbative Quantum Field Theory: Mathematical Aspects and Applications, Selected Papers (World Scientific, Singapore, 1992), pp. 523-545.

[3] H. Hamber and R.M. Williams, Nucl.Phys. B, 435, 361 (1995), hep-th/9406163.

[4] H. Hamber and R.M. Williams, Phys. Rev. D, 59, 064014 (1999), hep-th/9708019. 
[5] T. Regge and R.M. Williams, Journ. Math. Phys., 41, 3964 (2000), gr-qc/0012035.

[6] M. Caselle, A D'Adda and L. Magnea, Phys. Lett., 232B, 457 (1989).

[7] V.M. Khatsymovsky, Class. Quantum Grav., 6, L249 (1989).

[8] V.M. Khatsymovsky, Gen. Rel. Grav., 27, 583 (1995), gr-qc/9310004.

[9] V.M. Khatsymovsky, Class. Quantum Grav., 11, 2443 (1994), gr-qc/9310040.

[10] J.W. Barrett, M. Roček and R.M. Williams, Class. Quantum Grav., 16, 1373 (1999), gr-qc/9710056.

[11] V.M. Khatsymovsky, Phys. Lett., 560B, 245 (2003), gr-qc/0212110.

[12] H. Waelbroeck, Class. Quantum Grav., 7, 751 (1990).

[13] V.M. Khatsymovsky, Phys. Lett., 567B, 288 (2003), gr-qc/0304006.

[14] C. Wainwright and R.M. Williams, Class.Quantum Grav., 21, 4865 (2004), grqc/0405031.

[15] V.M. Khatsymovsky, Journ. Exp. Theor. Phys., 101, 420 (2005), gr-qc/0506071. 